\title{
Heterogeneity in Nucleosome Spacing Governs Chromatin Elasticity
}

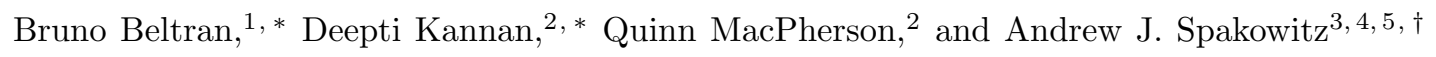 \\ ${ }^{1}$ Biophysics Program, Stanford University, Stanford, California 94305, USA \\ ${ }^{2}$ Department of Physics, Stanford University, Stanford, California 94305, USA \\ ${ }^{3}$ Chemical Engineering Department, Stanford University, Stanford, California 94305, USA \\ ${ }^{4}$ Department of Materials Science and Engineering, \\ Stanford University Stanford, California 94305, USA \\ ${ }^{5}$ Department of Applied Physics, Stanford University, Stanford, CA 94305
}

(Dated: July 19, 2019)

\begin{abstract}
Within a living cell, the myriad of proteins that bind DNA introduce heterogeneously spaced kinks into an otherwise semiflexible DNA double helix. To investigate the effects of heterogeneous nucleosome binding on chromatin organization, we extend the wormlike chain (WLC) model to include statistically spaced, rigid kinks. On time scales where nucleosome positions are fixed, we find that the probability of chromatin loop formation can differ by up to six orders of magnitude between two sets of nucleosome positions drawn from the same distribution. On longer time scales, we show that continuous re-randomization due to nucleosome turnover results in chromatin tracing out an effective WLC with a dramatically smaller Kuhn length than bare DNA. Together, these observations demonstrate that heterogeneity in nucleosome spacing acts as the dominant source of chromatin elasticity and governs both local and global chromatin organization.
\end{abstract}

PACS numbers: 05.20.-y, 05.40.Fb, 36.20.Ey, 87.10.Ca, 87.14.gk, 87.15-v, 87.16.Sr

The spatial organization of chromatin - genomic DNA and its associated proteins - is critical to a range of biological processes, from controlling gene expression [1] to facilitating DNA damage repair [2, 3]. The fundamental unit of eukaryotic chromatin organization is the nucleosome, which consists of 147 basepairs of DNA wrapped around a histone-protein octamer 44. Linker DNA connecting adjacent nucleosomes ranges from less than $10 \mathrm{bp}$ on average in fission yeast [5] to more than $80 \mathrm{bp}$ in sea urchin sperm cells [6].

In vitro images of chromatin have historically contained regular, "30-nm fiber" helical structures, motivating models of chromatin with constant (periodic) nucleosome spacing [7-16. However, recent super-resolution microscopy measurements indicate that in vivo, interphase mammalian chromatin instead forms a disordered, "beads-on-a-string" structure [17-19]. In addition, the latest in vivo nucleosome positioning data suggest that linker lengths are extremely heterogeneous. The occupancy profiles of even the most well-positioned nucleosomes - such as those near transcription start sites - are well described by a model where nucleosomes bind nearly uniformly along the DNA [11, 20,30] and are merely excluded from certain areas [31.

So far, works that address linker length heterogeneity have been either simulation studies [32, 33] or purely geometrical models [34, 35. These models can produce individual "beads-on-a-string" configurations qualitatively similar to those observed in bulk chromatin. However, there remains a need for an analytical approach that can systematically characterize how and when linker length heterogeneity leads to structural disorder.

In this paper, we characterize the structures that emerge from the combined effects of thermal fluctua- tions in the DNA linkers and the geometric effects of experimentally-relevant linker length heterogeneity. We show that almost any linker length heterogeneity is sufficient to produce the types of disordered chromatin structures that are now believed to dominate nuclear architecture. The intuition behind our structural claims extends to any polymer composed of aperiodic kinks, such as the dihedral "kinks" found at junctions of a block copolymer. More broadly, our results contribute to a large class of problems in which quenched disorder competes with thermal fluctuations to determine the structural properties of a system.

We model each DNA linker as a twistable wormlike chain (TWLC), and the nucleosomes as the points where these linker strands connect. At each nucleosome, we impose the constraint that the incoming and outgoing linker DNA strands have a fixed relative orientation. The orientation of the strand entering the $i$ th nucleosome is defined by the matrix $\Omega_{\text {entry }}^{(i)} \in S O(3)$ that rotates the lab frame into the entry orientation. The exit orientation $\Omega_{\text {exit }}^{(i)}$ is related to the entry orientation by a kink rotation $\Omega_{\text {kink }}$, such that $\Omega_{\text {exit }}^{(i)}=\Omega_{\text {entry }}^{(i)} \cdot \Omega_{\text {kink }}$, as shown in Fig. 1 .

We represent a TWLC of length $L$ as a space curve $\vec{R}(s), s \in[0, L]$. The chain orientation at each point along this curve, $\Omega(s)$, is represented by an orthonormal $\operatorname{triad} \vec{t}_{i}$, where $\vec{t}_{3}:=\partial_{s} \vec{R}(s)$. We track the bend and twist of our polymer via the angular "velocity" vector $\vec{\omega}(s)$, which operates as $\partial_{s} \vec{t}_{i}(s)=\vec{\omega}(s) \times \vec{t}_{i}(s)$. The Green's function of the first linker represents the probability that a polymer of length $L_{1}$ that begins at the origin with fixed initial orientation $\Omega_{0}$ ends at position $\vec{R}$ with fixed end orientation $\Omega$. For a TWLC with no kinks, the Green's 
function is given by

$$
G_{\mathrm{TWLC}}\left(\vec{R}, \Omega \mid \Omega_{0} ; L_{1}\right)=\int_{\Omega(0)=\Omega_{0}}^{\Omega(s)=\Omega} \mathscr{D}[\Omega(s)] e^{-\beta \mathcal{E}} \delta\left(\vec{R}-\int_{0}^{L_{1}} \overrightarrow{t_{3}} d s\right)
$$

where the energy

$$
\beta \mathcal{E}=\frac{l_{p}}{2} \int_{0}^{L_{1}} d s\left(\omega_{1}^{2}+\omega_{2}^{2}\right)+\frac{l_{t}}{2} \int_{0}^{L_{1}} d s\left(\omega_{3}-\tau\right)^{2}
$$

is quadratic in bending and twisting deformation. The natural twist of DNA gives $\tau=2 \pi(10.5 \mathrm{bp})^{-1}$, and we set the persistence length $l_{p}=50 \mathrm{~nm}$ and twist persistence length $l_{t}=100 \mathrm{~nm}$ to match measurements of DNA elasticity 36 39].

Reference [40, solves Equation 1]analytically in Fourier space $(\vec{R} \rightarrow \vec{k})$ by computing the coefficients in the Wigner D-function expansion

$$
\hat{G}_{\mathrm{TWLC}}\left(\vec{k}, \Omega \mid \Omega_{0} ; L_{1}\right)=\sum_{l ; l_{0} m_{0} j_{0}} g_{l_{0} m_{0} j_{0}}^{l m_{0} j_{0}} \mathcal{D}_{l}^{m_{0} j_{0}}(\Omega) \mathcal{D}_{l_{0}}^{m_{0} j_{0} *}\left(\Omega_{0}\right) .
$$

To account for the kink introduced by the nucleosome, we rotate the final orientation of the linker DNA, $\Omega=\Omega_{\text {entry }}$, to $\Omega_{\text {exit }}$ using the formula

$$
\mathcal{D}_{l}^{m_{0} j_{0}}\left(\Omega \cdot \Omega_{\text {kink }}\right)=\sum_{j} \sqrt{\frac{8 \pi}{2 l+1}} \mathcal{D}_{l}^{m_{0} j}\left(\Omega_{\text {kink }}\right) \mathcal{D}_{l}^{j j_{0}}(\Omega) .
$$

The resulting Green's function combines the effects of a linker DNA and a nucleosome, but is still a Wigner D-function expansion with modified coefficients $B_{l_{0} m_{0} j_{0}}^{l m_{0} j}$ (first computed in Ref. 41]). We present an alternative derivation in Supplemental Material [42].

X-ray crystallography [4, 44, 45] and cryo-EM [43, 4649] measurements of the nucleosome show that histonebound DNA is well approximated by a deformed B-DNA structure, wrapping the histone octamer 1.7 times in a superhelix with a radius of $4.19 \mathrm{~nm}$ and a pitch of $2.59 \mathrm{~nm}$ [45. Thus, $\Omega_{\text {entry }}$ and $\Omega_{\text {exit }}$ are well defined as a function of the number of bound nucleotides to the histone core. In what follows, we fix the wrapping level to that found in the crystal structure (147 bp). Using different values for the wrapping level rescales our results (see Supplemental Material [42, Fig. S3 and Fig. S10).

To compose monomers of the nucleosome chain with prescribed linker lengths, we perform an iterated convolution of the Green's function for each nucleosome-linker pair. In Fourier space, this corresponds to multiplying the matrices $B_{l_{0} m_{0} j_{0}}^{l m_{0} j}\left(L_{i}\right)$.

A key property of our model is that the relative orientation of adjacent nucleosomes is not only determined by $\Omega_{\text {kink }}$ and the thermal fluctuations of the linker strand, but also by changing the length of the linker strand (as demonstrated in Fig. 1b). Our propagator $G$ takes this into account implicitly due to the inclusion of $\tau$ in Eq. 2 .

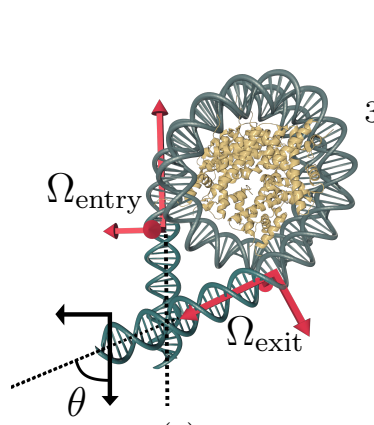

(a)

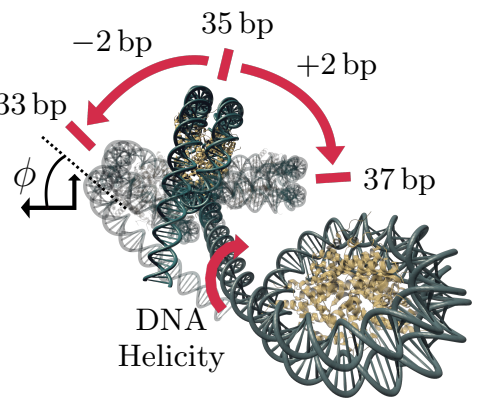

(b)
FIG. 1. (a) The structure of a human nucleosome 43 with straight linkers extrapolated from the entry $\left(\Omega_{\text {entry }}\right)$ and exit $\left(\Omega_{\text {exit }}\right)$ orientations of the bound DNA. The amount of DNA wrapping the nucleosome dictates the spherical angle $\theta$. (b) Two adjacent nucleosomes at zero temperature. The DNA double helix has an intrinsic twist density $(\tau=2 \pi /(10.5 \mathrm{bp}))$. If we anchor the location of one nucleosome, the binding orientation of the next histone octamer must change so that it aligns with the major groove of the double helix. This means that as the linker length $L$ connecting two nucleosomes gets longer or shorter, the relative orientations of adjacent octamers changes to create an angle $\phi=\tau L$.

We begin by computing the end-to-end distance $\sqrt{\left\langle R^{2}\right\rangle}$ of the chain using the formula $\lim _{k \rightarrow 0} \frac{\partial^{n} B_{000}^{000}}{\partial k^{n}}=$ $i^{n}\left\langle R^{n}\right\rangle$. From this, we extract the Kuhn length, $b=$ $\lim _{N \rightarrow \infty}\left\langle R^{2}\right\rangle / \sum_{i=0}^{N} L_{i}$, which gives a universal measure of the elasticity of a polymer at long length scales.

In Fig. 2a. we plot $\sqrt{\left\langle R^{2}\right\rangle}$ as a function of chain length for homogeneous chains of nucleosomes with $36 \mathrm{bp}$ and $38 \mathrm{bp}$ linkers. We compare each of these curves to the $\sqrt{\left\langle R^{2}\right\rangle}$ of a TWLC with the same Kuhn length but without kinks. At short length scales, the initial slope of $\sqrt{\left\langle R^{2}\right\rangle}$ for all chains is one, corresponding to rigid-rod behavior. At chain lengths comparable to the persistence length, the bare WLC's slope smoothly transitions to $1 / 2$ (on a log-log scale), corresponding to random-walk behavior. In contrast, the homogeneous chain $\sqrt{\left\langle R^{2}\right\rangle}$ jumps from that of bare DNA to that of the best-fit WLC, whose Kuhn length is dramatically smaller than twice the persistence length of bare DNA.

To build a geometric intuition for how the kinks create this modified Kuhn length, we compare the Kuhn lengths of homogeneous, fluctuating chains to their zerotemperature configurations, where the entire chain is composed of rigid-rod linkers. Every homogeneous chain at zero temperature forms a helix of nucleosomes. The rise per basepair of the helix is determined by the spherical angles $\theta$ and $\phi$ connecting adjacent linkers (see Fig. 1). The nucleosome structure fixes $\theta$, but $\phi$ depends linearly on the linker length, and is $10.5 \mathrm{bp}$ periodic due to the DNA's helicity. Select values of $\phi$ lead to more compact zero-temperature structures with a smaller rise per basepair. As seen in Figure 2b, the corresponding fluctuating structures have smaller Kuhn lengths, and those with a 


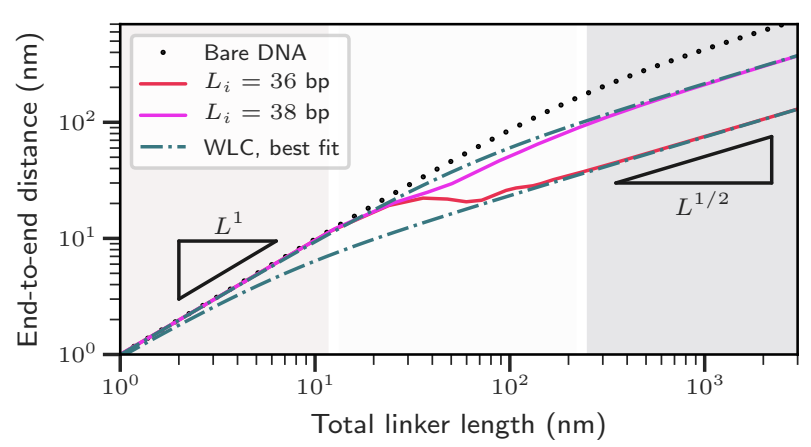

(a)

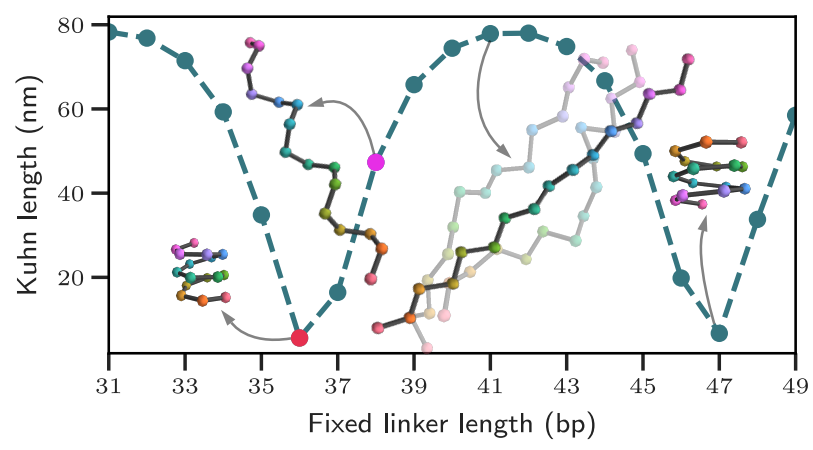

(b)

FIG. 2. (a) Average end-to-end distances for homogeneous chromatin chains with $36 \mathrm{bp}$ and $38 \mathrm{bp}$ linkers, compared to bare DNA and the best-fit WLCs. Rigid rod, Gaussian chain, and cross-over regimes highlighted. (b) Kuhn lengths of homogeneous chromatin chains are $10.5 \mathrm{bp}$ periodic in linker length. Example minimum energy chain configurations are shown, including two example Monte Carlo (fluctuating) structures for the $41 \mathrm{bp}$ case. Compact structures (36, $47 \mathrm{bp})$ afford more flexibility than less compact structures (41 bp).

larger rise per basepair at zero temperature have larger Kuhn lengths. The $10.5 \mathrm{bp}$ periodicity of $\phi$ as linker length changes leads to the periodicity in Figure $2 \mathrm{~b}$, As $L_{i} \rightarrow \infty$, the Kuhn length approaches that of bare DNA only slowly (see Supplemental Material [42, Fig. S4).

We next consider heterogeneous chains where the linker lengths are drawn uniformly from a range $\mu \pm \sigma$. In Fig. 3, we see that as we increase $\sigma$, the zero-temperature configuration of the chain interpolates between a helix at $\sigma=0$ and a random walk at larger $\sigma$. As a result, the zero-temperature structure itself has a Kuhn length, which describes the compactness of the random walk. As in the homogeneous case, the Kuhn length of the zero-temperature chain qualitatively predicts that of the fluctuating structure, as seen in Fig. 3 We find that even a single basepair of variance in nucleosome positions (see e.g. Supplemental Material [42, Fig. S5) can create enough geometric stochasticity at zero-temperature to prevent the formation of regular fibers.

In the cell, the simplest model of nucleosome binding is

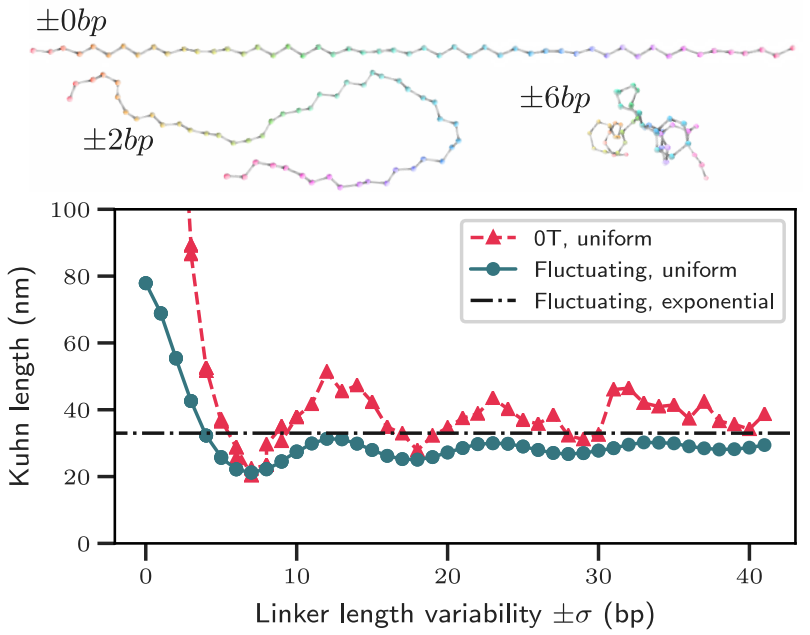

FIG. 3. Kuhn length of a heterogeneous chromatin chain with uniformly distributed linker lengths chosen from the range $\mu \pm \sigma$, where $\mu=41 \mathrm{bp}$. Example zero-temperature (0T) chains composed of rigid rod linkers are shown for $\sigma=0,2,6 \mathrm{bp}$. Kuhn length rapidly approaches that of the exponential chain (black line) in which linker lengths are exponentially distributed about the same $\mu$.

one in which nucleosomes bind uniformly randomly along the DNA [23. In this model, the distances separating the nucleosomes are exponentially distributed (hereafter "the exponential chain"). While this picture ignores some details of in vivo nucleosome formation, Fig. 3 shows how any linker length distribution with sufficiently large variance $(\sigma)$ will exhibit behavior similar to the exponential chain. Thus, the results that follow are likely robust to adding more detail to the nucleosome binding model.

When averaged over the distribution of possible nucleosome positions, the end-to-end distance of our exponential chain takes the form of a WLC with a rescaled Kuhn length (see Supplemental Material [42, Fig. S6). This is not wholly unsurprising, since at zero temperature our model differs from a freely rotating chain only in the correlation between linker length and $\phi$, and the freely rotating chain is known to converge to a wormlike chain under appropriate scaling [50]. We extract the rescaled Kuhn length as a function of $\left\langle L_{i}\right\rangle$ in Fig. 4

Unlike the homogeneous case, where changing $L_{i}$ selects between zero-temperature helices, increasing $\left\langle L_{i}\right\rangle$ in the heterogeneous case scales the zero-temperature random walk. As a result, Fig. 4 lacks the $10.5 \mathrm{bp}$ periodicity of the homogeneous chain. Thus, for the purposes of coarse-graining, an approximate knowledge of $\left\langle L_{i}\right\rangle$ should be sufficient to capture chromatin's average behavior as a WLC. A table of these Kuhn lengths is available in the Supplemental Material [42, Table S1.

While the Kuhn length describes the chain's elasticity on long length scales, a more useful metric on shorter length scales is the probability of genomic contacts (i.e. 


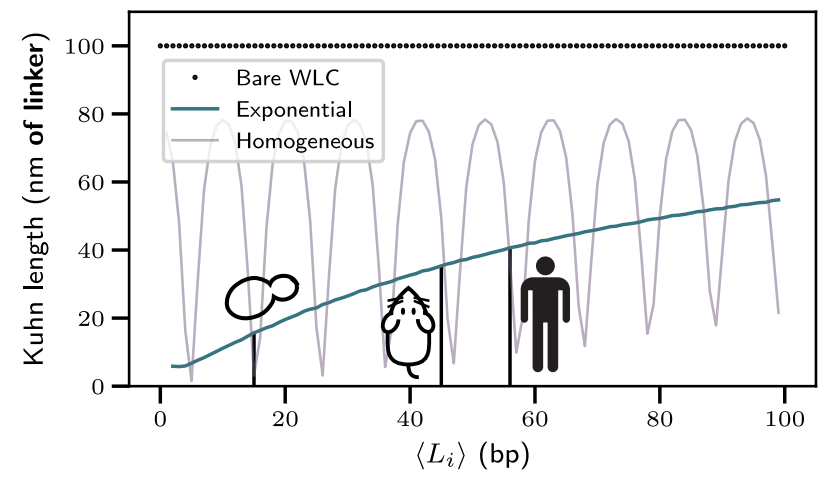

FIG. 4. Kuhn lengths of chromatin chains with exponentially distributed linkers, as a function of the average linker length, which varies by cell type. Kuhn lengths for $S$. cerevisiae $\left(\left\langle L_{i}\right\rangle=15 \mathrm{bp}\right)$, mice embryonic stem cells (45 bp), and human $\mathrm{T}$ cells (56 bp) are marked.

the looping probability). By numerically inverting the Fourier transform in Eq. 3, we can analytically evaluate $P_{\text {loop }}=G(\vec{R}=0 \mid L)$, a modified $J$-factor with no orientational component. In Fig. 5 , we plot this probability as a function of the loop size.

Strikingly, we observe that for a fixed genomic separation, the difference between the most- and leastlikely to loop chains spans up to six orders of magnitude depending on the intervening nucleosomes' spacings. The average looping probability, which is relevant at timescales where nucleosome positions can re-randomize, is captured well by a single WLC. Due to this effective WLC's reduced Kuhn length, we predict that chromatin's propensity for forming sub-kilobase-scale loops should be one to four orders of magnitude larger than that of bare DNA. The predicted looping propensity peaks at a length scale typical of promoter contacts in vivo and consistent with Hi-C looping data [51] (see Supplemental Material 42, Fig. S8). This result highlights how even without models of DNA more detailed than the WLCfor example those including DNA melting [52, 54 - the propensity of small DNA loops can be enhanced by proteins that promote kinks in the DNA if they are stochastically spaced.

Additionally, we find that the average looping probability is higher than that of most of the individual chains. Thus, even an "informationless" chromatin remodeler that merely promotes random nucleosome repositioning will greatly facilitate sub-kilobase loop formation. At longer length scales, the looping probabilities approach the characteristic $L^{-3 / 2}$ Gaussian scaling. However, individual chains retain memory of their kinks, as indicated by how the highlighted chains persist above or below the average. Using other linker length distributions, such as uniformly distributed linkers, leads to qualitatively similar results (see Supplemental Materials [42, Fig. S9).

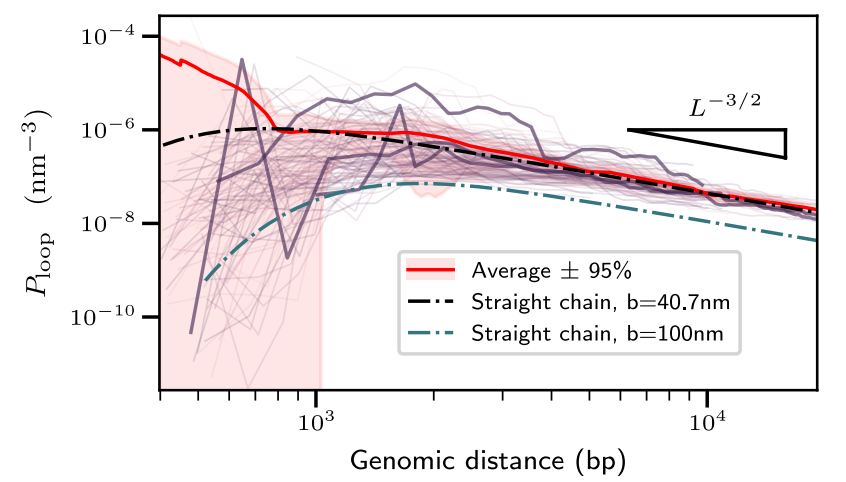

FIG. 5. Looping probabilities as a function of genomic separation for exponential chains. Each purple line designates an individual chain with linker lengths drawn from an exponential distribution with $\mu=56 \mathrm{bp}$. The red shaded area corresponds to $95 \%$ confidence intervals around the mean over the individual chains (red line). A wormlike chain (black dashed line) with the same Kuhn length as this average chain captures the looping probability for loops with at least three intervening nucleosomes. Three random individual chains are bolded.

In conclusion, we provide rigorous justification for using an effective WLC to model in vivo chromatin. Due to the lack of experimental consensus on the persistence length $l_{p}$ of chromatin [55, coarse-grained models of chromatin have historically used a range of values for $l_{p}$, sometimes even just using that of bare DNA [56 58 . We show that this choice leads to at least a two-fold overestimation of the polymer's stiffness and a several orders-of-magnitude underestimation of looping at short length scales. Some past models have extracted a parameter that describes the linear compaction of chromatin (bp per nm of fiber) from Hi-C looping probabilities (see e.g. [59, 60]). Our model's parameter-free estimate of chromatin's stiffness provides a theoretical explanation for the effective Kuhn length predicted from these experimental measurements.

Our model excludes various important facets of chromatin's structure, such as interaction energies (sterics and stacking) and nucleosome "breathing". Since nucleosome breathing simply corresponds to choosing a different distribution for the angle $\theta \in[0, \pi]$ between adjacent nucleosomes, incorporating breathing leaves our results qualitatively unchanged (see Supplemental Material [42, Fig. S10). However, a careful inclusion of breathing will likely require an explicit treatment of the effects of DNA sequence and linker histone on the nucleosome particle.

Our work highlights that the geometric effects of heterogeneous nucleosome binding dominate thermal fluctuations in determining chromatin's elasticity in vivo. Our insights into chromatin's stiffness will also inform future studies on the effects of loop extrusion factors 61 and epigenetic states 62] on chromatin organization.

Financial support for this work is provided by the Na- 
tional Science Foundation (NSF), Physics of Living Systems Program (PHY-1707751). Q.M. and B.B. acknowledge funding support from the NSF Graduate Fellowship program (DGE-1656518). B.B. acknowledges support from NIH Training Grant T32GM008294.

* These authors contributed equally to this work.

† ajspakow@stanford.edu

[1] M. R. Hübner, M. A. Eckersley-Maslin, and D. L. Spector, Curr Opin Genet Dev 23, 89 (2013).

[2] M. H. Hauer and S. M. Gasser, Genes Dev. 31, 2204 (2017)

[3] J. Stadler and H. Richly, Int J Mol Sci 18 (2017), 10.3390/ijms18081715

[4] A. Cutter and J. J. Hayes, FEBS Lett 589, 2914 (2015)

[5] R. M. Givens, W. K. M. Lai, J. M. Rizzo, J. E. Bard, P. A. Mieczkowski, J. Leatherwood, J. A. Huberman, and M. J. Buck, Nucleic Acids Res 40, 7176 (2012)

[6] C. Spadafora, M. Bellard, J. Lee Compton, and P. Chambon, FEBS Letters 69, 281 (1976).

[7] S. A. Grigoryev, G. Bascom, J. M. Buckwalter, M. B. Schubert, C. L. Woodcock, and T. Schlick, PNAS 113, 1238 (2016)

[8] N. Kepper, D. Foethke, R. Stehr, G. Wedemann, and K. Rippe, Biophysical Journal 95, 3692 (2008)

[9] E. F. Koslover, C. J. Fuller, A. F. Straight, and A. J. Spakowitz, Biophysical Journal 99, 3941 (2010)

[10] J. Langowski and D. W. Heermann, Seminars in Cell \& Developmental Biology Membrane Lipid Microdomains: Roles in Signalling and Disease and 3D Chromatin, 18, 659 (2007)

[11] O. Müller, N. Kepper, R. Schöpflin, R. Ettig, K. Rippe, and G. Wedemann, Biophysical Journal 107, 2141 (2014)

[12] H. Schiessel, W. M. Gelbart, and R. Bruinsma, Biophys J 80, 1940 (2001).

[13] A. Scipioni, G. Turchetti, S. Morosetti, and P. De Santis, Biophysical Chemistry 148, 56 (2010).

[14] G. Wedemann and J. Langowski, Biophysical Journal 82, $2847(2002)$

[15] G. D. Bascom and T. Schlick, in Nuclear Architecture and Dynamics, Translational Epigenetics, Vol. 2, edited by C. Lavelle and J.-M. Victor (Academic Press, Boston, 2018) pp. 123-147.

[16] E. Koslover and A. J. Spakowitz, Soft Matter 9, 7016 (2013)

[17] H. D. Ou, S. Phan, T. J. Deerinck, A. Thor, M. H. Ellisman, and C. C. O'Shea, Science 357, eaag0025 (2017).

[18] M. A. Ricci, C. Manzo, M. F. García-Parajo, M. Lakadamyali, and M. P. Cosma, Cell 160, 1145 (2015)

[19] T. Nozaki, R. Imai, M. Tanbo, R. Nagashima, S. Tamura, T. Tani, Y. Joti, M. Tomita, K. Hibino, M. T. Kanemaki, K. S. Wendt, Y. Okada, T. Nagai, and K. Maeshima, Molecular Cell 67, 282 (2017)

[20] R. D. Kornberg and L. Stryer, Nucleic Acids Res 16, 6677 (1988).

[21] G. Chevereau, L. Palmeira, C. Thermes, A. Arneodo, and C. Vaillant, Physical Review Letters 103 (2009), 10.1103/PhysRevLett.103.188103
[22] R. V. Chereji and A. V. Morozov, Journal of Statistical Physics 144, 379 (2011).

[23] D. A. Beshnova, A. G. Cherstvy, Y. Vainshtein, and V. B. Teif, PLOS Computational Biology 10, e1003698 (2014)

[24] T. Chou, Physical Review Letters 99 (2007), 10.1103/PhysRevLett.99.058105.

[25] R. Kornberg, Nature 292, 579 (1981)

[26] T. N. Mavrich, I. P. Ioshikhes, B. J. Venters, C. Jiang, L. P. Tomsho, J. Qi, S. C. Schuster, I. Albert, and B. F. Pugh, Genome Res. 18, 1073 (2008).

[27] W. Möbius and U. Gerland, PLoS Comput. Biol. 6 (2010), 10.1371/journal.pcbi.1000891

[28] W. Möbius, B. Osberg, A. M. Tsankov, O. J. Rando, and U. Gerland, PNAS 110, 5719 (2013).

[29] V. B. Teif and K. Rippe, Journal of Physics: Condensed Matter 22, 414105 (2010)

[30] S. Tesoro, I. Ali, A. N. Morozov, N. Sulaiman, and D. Marenduzzo, Phys. Biol. 13, 016004 (2016)

[31] E. A. Ozonov and E. van Nimwegen, PLOS Computational Biology 9, e1003181 (2013).

[32] R. Collepardo-Guevara and T. Schlick, PNAS 111, 8061 (2014)

[33] G. D. Bascom, T. Kim, and T. Schlick, The Journal of Physical Chemistry B 121, 3882 (2017)

[34] S. A. Grigoryev and L. B. Ioffe, FEBS Letters 130, 43 (1981).

[35] C. L. Woodcock, S. A. Grigoryev, R. A. Horowitz, and N. Whitaker, PNAS 90, 9021 (1993)

[36] P. J. Hagerman, Annual Review of Biophysics and Biophysical Chemistry 17, 265 (1988)

[37] C. Bustamante, J. F. Marko, E. D. Siggia, and S. Smith, Science 265, 1599 (1994)

[38] Z. Bryant, M. D. Stone, J. Gore, S. B. Smith, N. R. Cozzarelli, and C. Bustamante, Nature 424, 338 (2003)

[39] J. F. Marko and E. D. Siggia, Macromolecules 28, 8759 (1995)

[40] A. J. Spakowitz, Europhysics Letters (EPL) 73, 684 (2006).

[41] Y. Zhou and G. S. Chirikjian, The Journal of Chemical Physics 119, 4962 (2003)

[42] (2019), see Supplemental Material at https:// www.overleaf.com/project/5bf089c8f9256e52006d9a4d for detailed derivation of $B_{l_{0} m_{0} j_{0}}^{l m j}$, validation of numerical methods, examples of different unwrapping levels, and tables of effective Kuhn lengths.

[43] M. Wakamori, Y. Fujii, N. Suka, M. Shirouzu, K. Sakamoto, T. Umehara, and S. Yokoyama, Scientific Reports 5, 17204 (2015)

[44] C. L. White, R. K. Suto, and K. Luger, EMBO J 20, 5207 (2001)

[45] T. J. Richmond and C. A. Davey, Nature 423, 145 (2003).

[46] J. Bednar, I. Garcia-Saez, R. Boopathi, A. R. Cutter, G. Papai, A. Reymer, S. H. Syed, I. N. Lone, O. Tonchev, C. Crucifix, H. Menoni, C. Papin, D. A. Skoufias, H. Kurumizaka, R. Lavery, A. Hamiche, J. J. Hayes, P. Schultz, D. Angelov, C. Petosa, and S. Dimitrov, Molecular Cell 66, 384 (2017).

[47] S. Bilokapic, M. Strauss, and M. Halic, Scientific Reports 8, 7046 (2018)

[48] M. Eltsov, D. Grewe, N. Lemercier, A. Frangakis, F. Livolant, and A. Leforestier, Nucleic Acids Research 46, 9189 (2018). 
[49] B.-R. Zhou, J. Jiang, H. Feng, R. Ghirlando, T. S. Xiao, and Y. Bai, Molecular Cell 59, 628 (2015).

[50] P. Kilanowski, P. March, and M. Samara, arXiv:1711.04032 [math] (2017), arXiv:1711.04032 [math]

[51] A. L. Sanborn, S. S. P. Rao, S.-C. Huang, N. C. Durand, M. H. Huntley, A. I. Jewett, I. D. Bochkov, D. Chinnappan, A. Cutkosky, J. Li, K. P. Geeting, A. Gnirke, A. Melnikov, D. McKenna, E. K. Stamenova, E. S. Lander, and E. L. Aiden, PNAS 112, E6456 (2015)

[52] J. Shimada and H. Yamakawa, Macromolecules 17, 689 (1984)

[53] Y. Liu, T. Pérez, W. Li, J. D. Gunton, and A. Green, The Journal of Chemical Physics 134, 065107 (2011)

[54] P. A. Wiggins, R. Phillips, and P. C. Nelson, Phys Rev E Stat Nonlin Soft Matter Phys 71, 021909 (2005).
[55] J. Langowski, The European Physical Journal E 19, 241 (2006)

[56] F. Benedetti, D. Racko, J. Dorier, Y. Burnier, and A. Stasiak, Nucleic Acids Res 45, 9850 (2017).

[57] Q. MacPherson, B. Beltran, and A. J. Spakowitz, PNAS , 201812268 (2018)

[58] J. Nuebler, G. Fudenberg, M. Imakaev, N. Abdennur, and L. A. Mirny, PNAS 115, E6697 (2018)

[59] S. K. Ghosh and D. Jost, PLoS Comput. Biol. 14, e1006159 (2018).

[60] A. Rosa and R. Everaers, PLoS Comput. Biol. 4, e1000153 (2008).

[61] C. A. Brackley, J. Johnson, D. Michieletto, A. N. Morozov, M. Nicodemi, P. R. Cook, and D. Marenduzzo, Phys. Rev. Lett. 119, 138101 (2017)

[62] D. Michieletto, E. Orlandini, and D. Marenduzzo, Physical Review X 6 (2016), 10.1103/PhysRevX.6.041047. 